\title{
Is there possibility of vertical transmission of COVID-19: a systematic review
}

\author{
Jiajun Yuan ${ }^{1,2 \#}$, Han Qian ${ }^{3 \#}$, Shiyan Cao ${ }^{4 \#}$, Bin Dong ${ }^{1}$, Xiaoyu Yan ${ }^{5}$, Shuanghong Luo ${ }^{6}$, Min Zhou ${ }^{1}$, \\ Sha Zhou ${ }^{7}$, Botao Ning ${ }^{1,3}$, Liebin Zhao ${ }^{1,2,8}$
}

${ }^{1}$ Shanghai Children's Medical Center, School of Medicine, Shanghai Jiao Tong University, Shanghai, China; ${ }^{2}$ Children Health Advocacy Institute, China Hospital Development Institute of Shanghai Jiao Tong University, Shanghai, China; ${ }^{3}$ Department of Pediatric Intensive Care Unit, Shanghai Children's Medical Center, School of Medicine, Shanghai Jiao Tong University, Shanghai, China; ${ }^{4}$ Department of Pediatrics, Changhai Hospital, Naval Medical University, Shanghai, China; ${ }^{5}$ Shanghai Institute of Hematology, Ruijin Hospital, School of Medicine, Shanghai Jiao Tong University, Shanghai, China; ${ }^{6}$ Department of Pediatrics, West China Second University Hospital, Sichuan University, Chengdu, China; ${ }^{7}$ Department of Hospital Infection Control, Shanghai Children's Medical Center, School of Medicine, Shanghai Jiao Tong University, Shanghai, China; ${ }^{8}$ School of Public Health, School of Medicine, Shanghai Jiao Tong University, Shanghai, China

Contributions: (I) Conception and design: B Ning, L Zhao; (II) Administrative support: B Ning, L Zhao; (III) Provision of study materials or patients: B Ning; (IV) Collection and assembly of data: H Qian, J Yuan, S Cao; (V) Data analysis and interpretation: J Yuan, H Qian, S Cao; (VI) Manuscript writing: All authors; (VII) Final approval of manuscript: All authors.

\#These authors contributed equally to this work.

Correspondence to: Liebin Zhao and Botao Ning. Shanghai Children's Medical Center, Shanghai Jiao Tong University School of Medicine, 1678 Dongfang Road, Shanghai 200127, China. Email: zhaoliebin@scmc.com.cn and ningbotao@126.com.

\begin{abstract}
In order to investigate the clinical features of pregnant women and their neonates with coronavirus disease 2019 (COVID-19) and the evidence of vertical transmission of COVID-19, we retrieved studies included in PubMed, Medline and Chinese databases from January 1, 2000 to October 25, 2020 using relevant terms, such as 'COVID-19', 'vertical transmission' et al. in 'Title/Abstract'. Case reports and case series were included according to the inclusion and exclusion criteria. We conducted literature screening and data extraction, and performed literature bias risk assessment. Total of 13 case series and 16 case reports were collected, including a total of 564 pregnant women with COVID-19 and their 555 neonates, of which 549 neonates received nucleic acid test for the severe acute respiratory syndrome coronavirus 2 (SARS-CoV-2) and 18 neonates was diagnosed with COVID-19. The positive rate is $3.28 \%$. Amniotic fluid of one woman was tested positive for SARS-CoV-2. The majority of infected neonates were born under strict infection control and received isolation and artificial feeding. Up till now, there is no sufficient evidence to exclude the possibility of vertical transmission for COVID-19 based on the current available data.
\end{abstract}

Keywords: Coronavirus disease 2019 (COVID-19); severe acute respiratory syndrome coronavirus 2 (SARS-

$\mathrm{CoV}-2)$; pregnant women; vertical transmission; neonates

Submitted May 21, 2020. Accepted for publication Dec 04, 2020.

doi: $10.21037 / \mathrm{tp}-20-144$

View this article at: http://dx.doi.org/10.21037/tp-20-144

\section{Introduction}

From the later of 2019, the severe acute respiratory syndrome coronavirus (SARS-CoV-2) spread rapidly from person to person around the world. Up till now, more than 50 million patients have been diagnosed as coronavirus disease 2019 (COVID-19) and more than 1million and 20 thousand patients died of it in almost all countries and regions. Due to the widespread of COVID-19 and its high mortality rate, shortage of medical resources has been occurring all over the world and threatening the public health. On February 11, 2020, the International 
Virus Classification Committee officially named the novel coronavirus as: the severe acute respiratory syndrome coronavirus 2 (SARS-CoV-2) (1). On February 12, 2020, the 2019 novel coronavirus pneumonia was officially named by the World Health Organization: COVID-19 (2).

The novel coronavirus pneumonia diagnosis and treatment program (trial version 8) released by the National Health Commission of China summarizes the transmission routes of COVID-19. Firstly, spreading via close-range respiratory droplets and close contact are the main transmission routes. Secondly, in a relatively closed environment, there may be aerosol transmission when exposed to high concentration aerosols for a long time. Thirdly, because novel coronavirus can be isolated in feces and urine, attention should be paid to the aerosol or contact transmission caused by environment polluted by feces and urine (3).

The interpersonal transmission of SARS-CoV and MERS-CoV, both belong to the Coronaviridae family, is also droplet transmission, and it mainly occurs in hospitals (4). Evidence suggests that compared to the general patient population, pregnant women with SARS and MERS are more likely to have serious complications and a higher risk of death from related events $(5,6)$. As the number of pregnant women and newborns infected with COVID-19 increased, people began to worry and look for evidence of mother-to-child transmission. There is no directed and sufficient evidence to show that SARS-CoV and MERS-CoV have vertical transmission. Similarly, there is currently insufficient evidence to show that there is vertical transmission of COVID-19.

However, it was reported that a neonate born to a mother with COVID-19 had elevated IgM antibody levels to SARS-CoV-2 in blood 2 hours after birth, which means COVID-19 could have vertical transmission potential (7). To summarize the relevant characteristics of COVID-19 pregnant women and analyzed the possibility of vertical transmission of COVID-19, we wrote this systematic review which included women who were infected with SARS-CoV-2 during pregnancy and their newborns during infection. This will help us actively respond to possible related infections in clinical work and protect the health of pregnant women and newborns suffering from COVID-19. We present the following article in accordance with the PRISMA reporting checklist (available at http://dx.doi.org/10.21037/tp-20-144).

\section{Methods}

\section{Inclusion criteria}

(I) Pregnant women who had positive results of nucleic acid test of SARS-CoV-2;

(II) The delivery during onset period;

(III) The onset of the neonates within 14 days of life;

(IV) Both Chinese and English documents that meet the standards.

\section{Database and retrieval time}

We searched PubMed, Medline, Chinese Biomedical Literature Database (CBM), Wanfang Database, and retrieval time was: January 1, 2000 to October 25, 2020.

\section{Literature retrieval strategy}

Relevant publications were retrieved from PubMed, Medline, CBM and Wanfang database through the following keywords or subject terms: COVID-19, vertical transmission, neonate and their similar or related words. Relevant references were traced. Here, we provided the search strategy in PubMed in detail as follows.

Search terms:

(I) COVID-19, severe acute respiratory syndrome coronavirus 2;

(II) COVID 19 virus, COVID-19 virus, SARS-CoV-2, coronavirus disease 2019 virus, SARS2, 2019-nCoV, 2019 novel coronavirus, 2019 novel coronavirus infection, COVID 19, coronavirus disease 2019, coronavirus disease-19, 2019-nCoV disease, 2019 novel coronavirus disease, $2019-\mathrm{nCoV}$ infection, novel coronavirus pneumonia;

(III) 'Infectious Disease Transmission, Vertical', maternalneonatal transmission, mother to child transmission, vertical transmission, perinatal infection;

(IV) Neonate, neonates, neonatus, newborn.

Search formula:

* \#1 ("COVID-19" [Supplementary Concept]) OR "severe acute respiratory syndrome coronavirus 2" [Supplementary Concept];

* \#2 ((()((COVID 19 virus [Title/Abstract]) OR SARS-CoV-2 [Title/Abstract]) AND COVID-19 virus [Title/Abstract]) OR coronavirus disease 2019 
virus [Title/Abstract]) OR SARS2 [Title/Abstract]) OR 2019-nCoV [Title/Abstract]) OR 2019 novel coronavirus [Title/Abstract]);

* \#3 $((((((2019$ novel coronavirus infection[Title/ Abstract]) OR COVID 19[Title/Abstract]) OR coronavirus disease 2019[Title/Abstract]) OR coronavirus disease-19[Title/Abstract]) OR 2019nCoV disease[Title/Abstract]) OR 2019 novel coronavirus disease[Title/Abstract]) OR 2019-nCoV infection[Title/Abstract]) OR novel coronavirus pneumonia[Title/Abstract];

* \#4 "Infectious Disease Transmission, Vertical"[Mesh];

* \#5 (((maternal-neonatal transmission) OR mother to child transmission) OR vertical transmission) OR perinatal infection;

* \#6(((Neonate [Title/Abstract]) OR neonates [Title/ Abstract]) OR neonates [Title/ Abstract]) OR newborn [Title/Abstract];

* \#7: \#1 OR \#2 OR \#3;

* \#8: \#4 OR \#5 OR \#6;

* \#9: \#7 AND \#8.

\section{Literature screening principles}

By reading the abstract, we excluded papers without abstracts, expert consensus, diagnosis and treatment plans, conference papers, reviews, lectures, studies in diagnostic reagents and molecular diagnosis. We re-screened by reading the full text to exclude, and eliminated documents that did not meet requirements according to inclusion criteria.

\section{Data extraction}

This system review mainly extracts three parts of data, including: basic data of pregnant women suffering from COVID-19 and their neonates and clinical data of newborns diagnosed with COVID-19. Variables extracted and analyzed included maternal age, gestational age at delivery, clinical characteristics and diagnostic methods of COVID-19. Results of SARS-CoV-2 laboratory tests of the neonates born to included mothers were also recorded. For SARS-CoV-2 positive neonates, we mainly extracted their diagnosis methods and time, infection control during and after delivery, the results of maternal examination materials that were tested for SARS-CoV-2 and so on.

Data extraction were done by one person and confirmed by another. Uncertain data were judged by the third one.

\section{Literature bias risk assessment}

Given that SARS-CoV-2 infection has a shorter epidemic time and fewer interventional studies, the literatures included in this study were all observational studies. The literature biased risk assessment tool for observational studies was selected through the Quality Appraisal Tool for Case Series (Institute of Health Economics) (8). The assessment was done both at the study and outcome level, and the protocol was described in detail in the tool mentioned.

\section{Statistical analysis}

Statistical analysis was done with SPSS for version 22.0 (IBM Corp.). Continuous variables were expressed as means and ranges. Categorical variables were expressed as numbers and percentages.

\section{Results}

\section{Literature search}

A total of 711 articles were retrieved in PubMed. 296 articles contained maternal-neonatal transmission and COVID-19 were transmitted in china biomedical (CBM) and Wanfang database. After screening, a total of 29 studies were included in the systematic review. Among them, 16 were case reports ( $\leq 3$ cases) $(7,9-23)$ and 13 were case series (24-36) (Figure 1).

\section{Literature bias risk assessment}

Of all the studies included in our systematic review, 13 were case series, and 16 were case reports (cases $\leq 3$ ). We used the case series research quality evaluation (IHE) tool to perform bias risk assessment. IHE has 20 items in 8 fields, and items that meet more than $70 \%$ means the risk of bias is acceptable. Six studies (28,31-35) satisfying more than $70 \%$ of the items are acceptable for the risks of bias, and the study of Oncel et al. (33) contained the most cases of maternal COVID-19. All studies satisfy 6 items (Item 2, 4, $6,10,17,18)$ absolutely while no study satisfy the Item 16 and 20 (Table 1).

\section{Characteristics of pregnant women with COVID-19}

A total of 564 pregnant women with COVID-19 were included in this systematic review (Table 2). Most of them were from China (43.44\%), America (34.04\%) and Turkey 


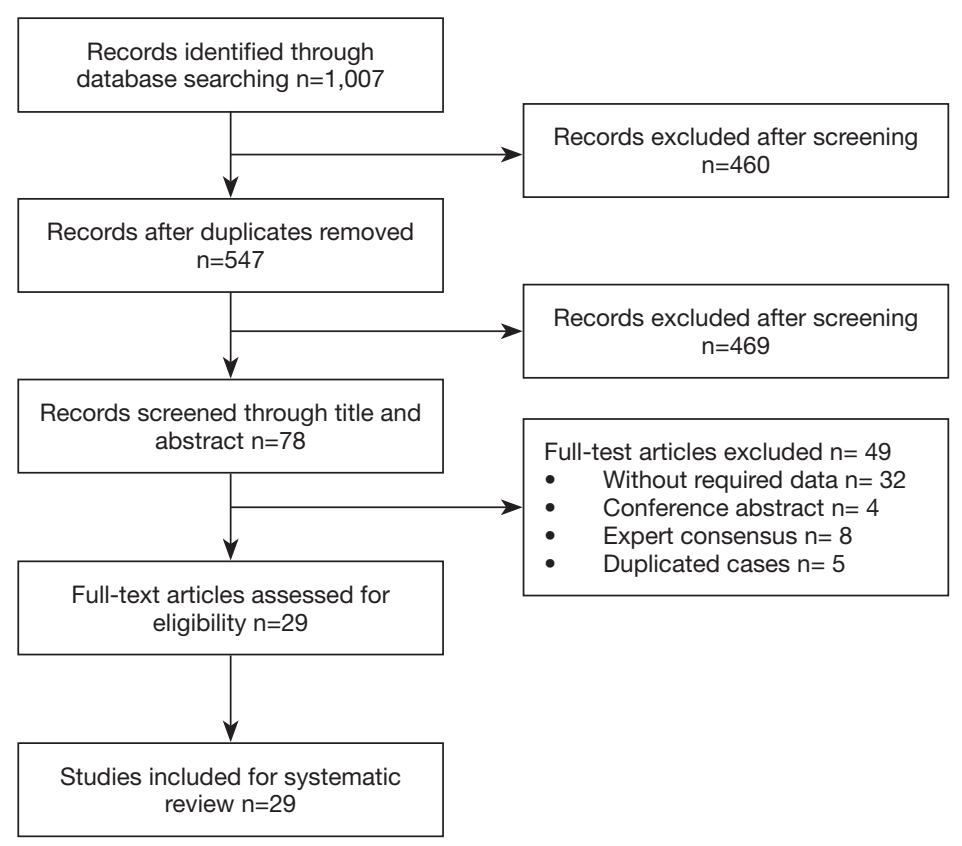

Figure 1 Literature screening process

(22.16\%). Except 238 women whose age was not reported, majority of the patients were found in the age group of $20-41$ years. Of all the 564 patients, $486(86.17 \%)$ were confirmed by positive results of SARS-CoV-2 RT-PCR, and the others were clinically diagnosed by clinical performance or chest CT. 121 (21.45\%) of all mothers reported never being symptomatic, and 318 (56.38\%) were symptomatic. Of all symptoms, fever was the most common with the incidence of $56.38 \%$, cough was the second. There were also some patients infected with the symptom of shortness of breath (16.04\%), myalgia (9.2\%) and gastrointestinal symptoms $(5.35 \%)$. Majority of the mothers had been pregnant for 30-41 weeks when admitted or confirmed with COVID-19 and more than half had symptoms onset average 2.95 days (with the range of $0-38$ days) before delivery. Only 98 (17.38\%) mothers received antiviral or antibiotic treatment before delivery. Among the 522 patients who delivered successfully, 104 (19.92\%) women delivered preterm and $328(62.84 \%)$ women delivered by caesarean. Besides, 135 (23.94\%) mothers were reported associated comorbidities during pregnancy.

\section{Clinical characteristics of neonates born to pregnant women with COVID-19}

A total of 555 neonates were included in our studies, including 231 boys and 192 girls, 132 (23.78\%) neonates were not reported their gender information (Table 3). Except 88 babies, the average birthweight of all babies was $2,470.38 \mathrm{~g}$, within the range of $1,830-2,691$ g. $67.03 \%$ of all the 555 neonates were born by cesarean section and 105 (18.92\%) were preterm. Almost all neonates (549, 98.92\%) received the test for SARS-CoV-2, in which 18 were tested positive as the rate is $3.28 \%$. By the way, $134(24.41 \%)$ cases tested maternal examination materials, such as amniotic fluid, placental tissue, vaginal secretions, breast milk, umbilical cord blood.

\section{Characteristics of COVID-19 neonates}

Eighteen neonates were diagnosed with COVID-19, and 10 were male. The clinical features of these neonates are presented in Table 4. Case 4, 6, 7, 9 and 10 were premature babies, the other 13 neonates were full-term. Only Case 10 was born by vaginal delivery. Majority of neonates were born with a normal Apgar index [7-10] and with normal birth weight $(2,500-4,000 \mathrm{~g})$, and the only two exception was Case 9 and 10, both were low-birthweight infants.

The infection prevention during delivery of only 12 babies were described. Case 14-18 were observed in the operating room unprotected for 30 minutes and not separated from their mothers immediately after delivery. 


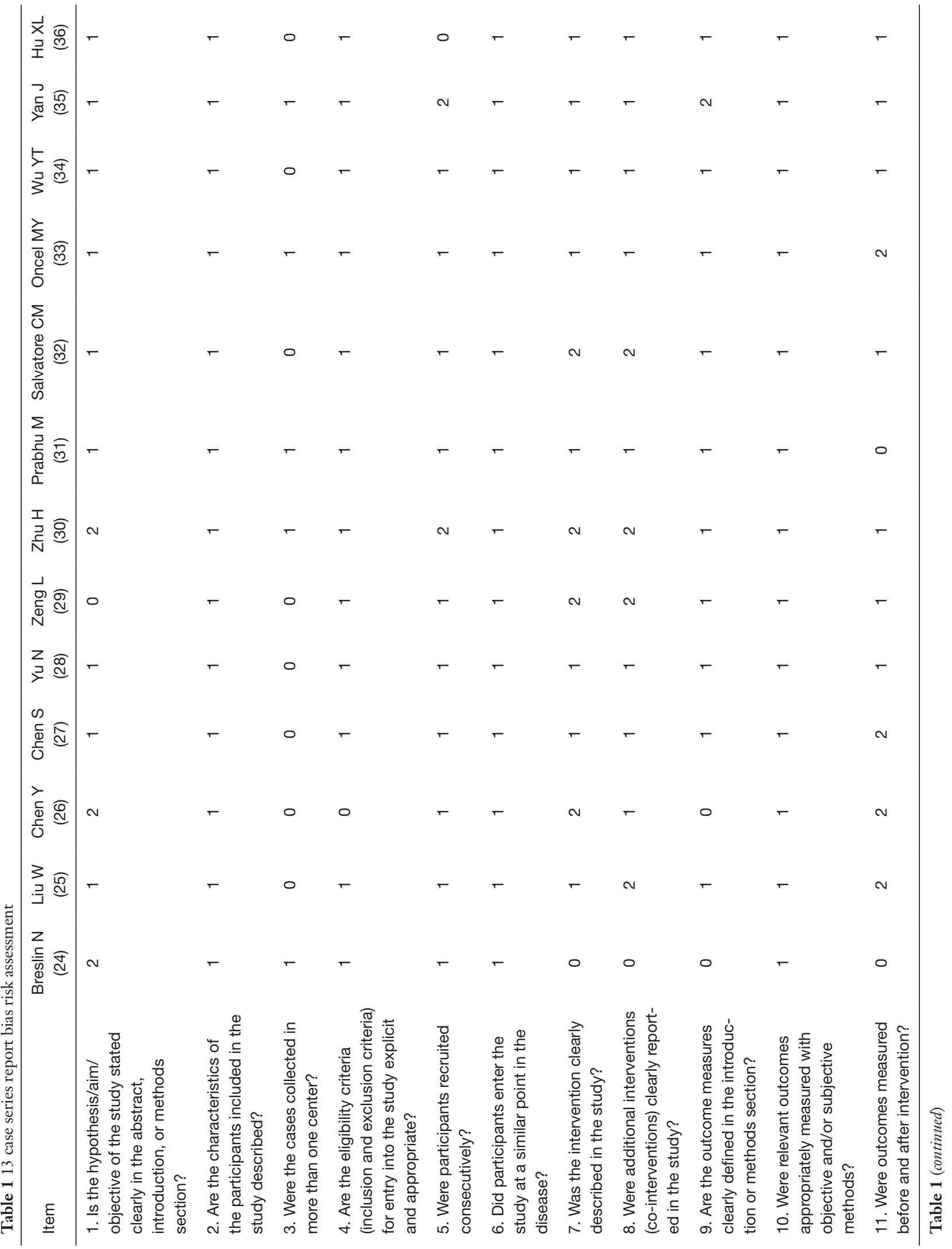




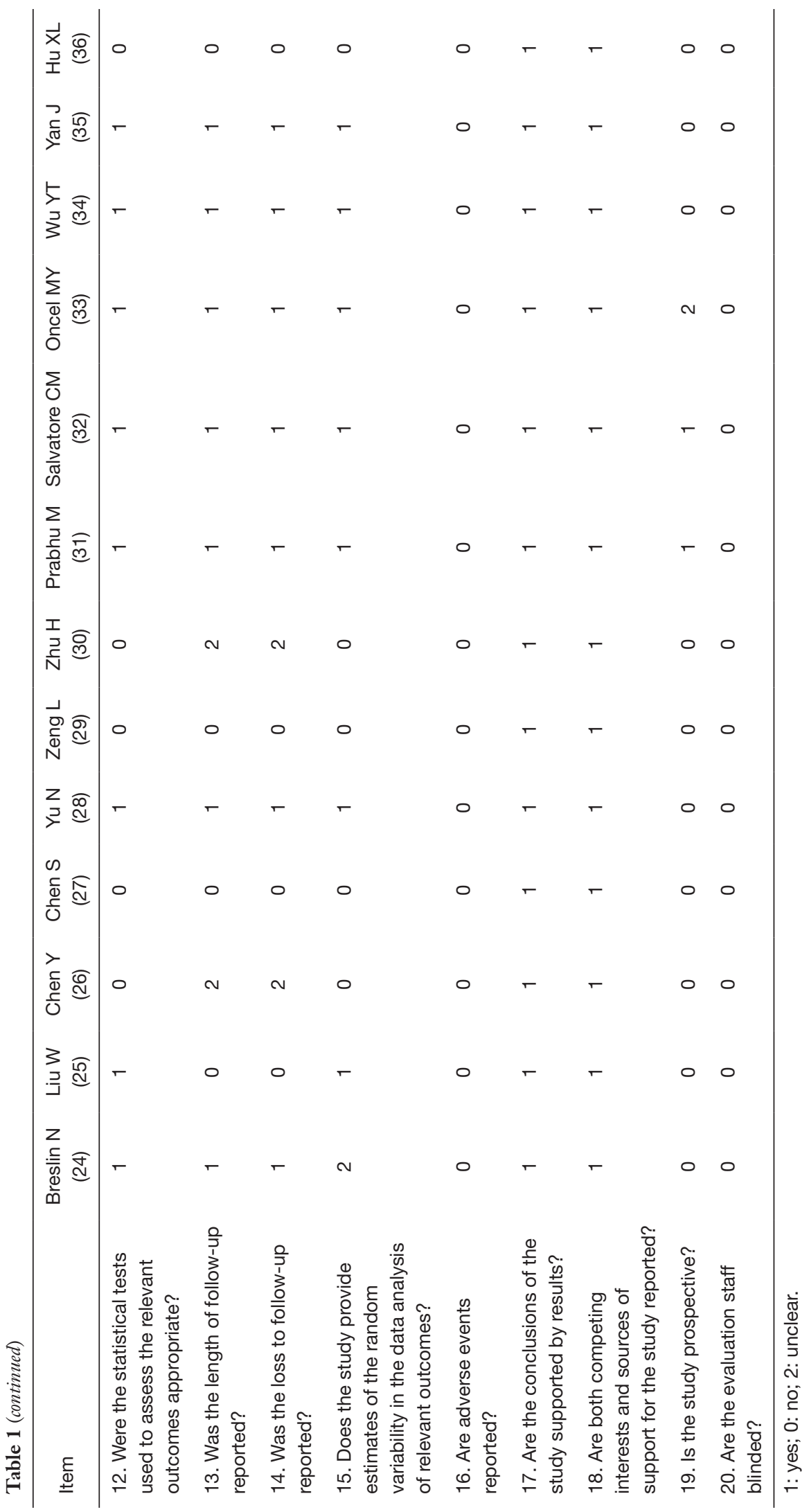


Table 2 Maternal characteristics

All mothers $(\mathrm{N}=564)$

Country
China
America
Turkey
Japan
Iran

Age, years

Not reported

$$
\begin{gathered}
245(43.44 \%) \\
192(34.04 \%) \\
125(22.16 \%) \\
1(0.02 \%) \\
1(0.02 \%) \\
30.21(20-41) \\
238(42.2 \%) \\
486(86.17 \%) \\
78(13.83 \%) \\
318(56.38 \%) \\
121(21.45 \%) \\
125(22.16 \%)
\end{gathered}
$$

Method of diagnosis

Laboratory confirmed (PCR)

Clinically diagnosed

Maternal symptoms

Symptomatic

Asymptomatic

Not reported

Type of maternal symptoms

Fever

180/318 (56.6\%)

Cough

$130 / 318(40.88 \%)$

Shortness of breath

$51 / 318(16.04 \%)$

Myalgia

29/318 (9.2\%)

Gastrointestinal

$17 / 318(5.35 \%)$

Gestational age at admission or diagnosis

$37.5(30-41)$

Not reported

$179(31.74 \%)$

Onset of diagnosis or symptoms to delivery, days

$2.95(0-38)$

Not reported

$257(45.57 \%)$

Treatment before delivery

$98(17.38 \%)$

Delivery

Giving birth

$522(92.55 \%)$

Preterm delivery (<37 weeks)

$104 / 522(19.92 \%)$

Mode of delivery

Caesarean delivery

$328 / 522(62.84 \%)$

Vaginal delivery

$116 / 522(22.22 \%)$

Not reported

$78 / 522(14.94 \%)$

Associated comorbidities

$135(23.94 \%)$

Not reported

$269(47.7 \%)$

$\mathrm{PCR}$, polymerase chain reaction. 
Table 3 Neonatal characteristics

\begin{tabular}{|c|c|}
\hline & All neonates $(\mathrm{N}=555)$ \\
\hline \multicolumn{2}{|l|}{ Gender } \\
\hline Male & $231(41.62 \%)$ \\
\hline Female & $192(34.59 \%)$ \\
\hline NR & $132(23.78 \%)$ \\
\hline Prematurity (<37 weeks) & $105(18.92 \%)$ \\
\hline Birthweight, grams & $2,470.38(1,830-2,691)$ \\
\hline NR & $88(15.86 \%)$ \\
\hline \multicolumn{2}{|l|}{ Mode of delivery } \\
\hline Cesarean delivery & $372(67.03 \%)$ \\
\hline Vaginal delivery & $182(32.79 \%)$ \\
\hline \multicolumn{2}{|l|}{ Apgar score } \\
\hline $1 \mathrm{~min}$ & $8.53(6-10)$ \\
\hline $5 \mathrm{~min}$ & $9.38(8-10)$ \\
\hline \multicolumn{2}{|l|}{ Tested for SARS-CoV-2 } \\
\hline Yes & $549(98.92 \%)$ \\
\hline No & $6(1.08 \%)$ \\
\hline \multicolumn{2}{|l|}{ Mode of testing } \\
\hline Neonatal examination materials & $549(100 \%)$ \\
\hline Maternal examination materials & $134(24.41 \%)$ \\
\hline \multicolumn{2}{|l|}{ Results of test } \\
\hline Negative & $536 / 549$ (97.63\%) \\
\hline Positive & $18 / 549(3.28 \%)$ \\
\hline
\end{tabular}

NR, not reported. SARS-CoV-2: the severe acute respiratory syndrome coronavirus 2. Neonatal examination materials: nasopharyngeal swabs, anal swabs, sputum sample, peripheral blood, urine, feces, serum antibodies (IgG and IgM for SARSCoV-2). Maternal examination materials: amniotic fluid, placental tissue, vaginal secretions, breast milk, umbilical cord blood.

The remaining neonates were under strict infection control during delivery and isolated immediately after birth. Case 5-7, 9-12 and 18 were fed with formula after birth, but the remaining cases did not report feeding methods. Mothers of two-thirds infants (Case1, 6-13 and 15-17) received antiviral or antibiotic treatment before delivery.

Except Case 10, 11, 13-17, all neonates were diagnosed within 3 days after birth. Most patients were diagnosed as confirmed COVID-19 infection based on the positive SARS-CoV-2 RNA test in neonatal examination samples, such as nasopharyngeal swabs, anal swabs, sputum, urine and feces samples. Although Case 8, 15 and 17 were tested negative for SARS-CoV-2, they had high level of IgG and IgM for SARS-CoV-2 in their serum, which also met the diagnostic criteria. Because of the characteristic COVID-19 pneumonia manifestations of both lungs (ground-glass opacity in bilateral peripheral lungs, Case 16 was also diagnosed as suspected COVID-19 infection. In addition, Case 1, 7, 8 and 18 also performed nucleic acid tests on samples of their mother's vaginal secretions, placenta, umbilical cord blood, amniotic fluid and breast milk. Except for the positive result of amniotic fluid in Case 7, the rest were negative.

Only case 2, 6 and 14 were reported symptoms, respectively, on the second day, sixth day and third day after birth. Besides, five infants had no symptoms. Only five cases described the prognosis of the neonates that their test for SARS-CoV-2 finally turned negative within 11 days of life.

\section{Discussion}

It has been controversial if there exists vertical transmission of COVID-19. Mother-to-child transmission includes vertical transmission in the womb, contact of the newborn with maternal blood and amniotic fluid during delivery, and postpartum infection, especially during breast feeding. In this systematic review, a total of 18 neonates were tested positive for SARS-CoV-2, thus the problem where were the sources and reasons of infection is worthy of attention.

SARS-CoV-2 infection in pregnancy has important effects on perinatal and neonatal outcomes. In our systematic review, pregnant women with COVID-19 have a higher rate of cesarean section but a normal rata of preterm delivery and comorbidities. We think this is because that the indications for caesarean delivery have been relaxed. However, we could not connect the high cesarean rate to the low positive rate of neonatal outcomes. Whether cesarean section could help protect the neonates from SARS-CoV-2 is still unknown and this question should be evaluated in future studies.

Most neonates were diagnosed with COVID-19 by positive SARS-CoV-2 RT-PCR test in nasopharyngeal swabs. According to New coronavirus pneumonia diagnosis and treatment program (Trial Version 8) issued by the National Health Commission of China (3), the positive results of SARS-CoV-2 RT-PCR is one of the diagnostic criteria. Most neonates were delivered under strict pollution control and were isolated timely after birth and accepted 
Table 4 Characteristics of neonates with COVID-19

\begin{tabular}{|c|c|c|c|c|c|c|c|c|c|c|c|c|c|c|c|c|c|}
\hline $\begin{array}{l}\text { Case } \\
\text { No. }\end{array}$ & Author & Country & Gender & Premature & Mode of delivery & Birthweight, grams & $\begin{array}{c}\text { Apgar at } 1 \text { and } \\
5 \mathrm{~min}\end{array}$ & $\begin{array}{l}\text { Infection } \\
\text { prevention }\end{array}$ & Separation & $\begin{array}{l}\text { Mode of } \\
\text { feeding }\end{array}$ & $\begin{array}{l}\text { Maternal } \\
\text { treatment }\end{array}$ & Diagnostic time & SARS-CoV-2 RT-PCR & Serum IgG and IgM & $\begin{array}{c}\text { Maternal examination } \\
\text { materials }\end{array}$ & Time of symptoms & Turn negative \\
\hline 1 & Yu N (28) & China & NR & No & $C D$ & 3,250 & 8,9 & NR & Yes & NR & Yes & $36 \mathrm{~h}$ & Unclear (+) & NR & PT and UCB (-) & NR & NR \\
\hline 2 & Zeng L (29) & China & Male & No & $C D$ & NR & NR & Strict $^{a}$ & Yes & NR & NR & $2 d$ & NPS and AS (+) & NR & NR & $2 d$ & $6 d$ \\
\hline 3 & Zeng L (29) & China & Male & No & $C D$ & NR & NR & Strict $^{a}$ & Yes & NR & NR & $2 d$ & NPS and AS (+) & NR & NR & NR & $6 \mathrm{~d}$ \\
\hline 4 & Zeng L (29) & China & Male & Yes $(31 w, 2 d)$ & $C D$ & NR & 3,4 & Strict $^{a}$ & Yes & NR & NR & $2 d$ & NPS and AS (+) & NR & NR & NR & $7 \mathrm{~d}$ \\
\hline 5 & Li MD (17) & China & Male & No & $C D$ & NR & NR & Strict $^{a}$ & Yes & FM & No & $3 d$ & NPS (+) & NR & NR & NR & NR \\
\hline 6 & Alzamora MC (20) & American & Male & Yes $(33 w)$ & $C D$ & 2,970 & 6,8 & Strict $^{a}$ & Yes & FM & Yes & $16 \mathrm{~h}$ & NPS (+) & NR & NR & $6 d$ & NR \\
\hline 7 & Zamaniyan M (21) & Iran & Female & Yes (32w) & $C D$ & 2,350 & 8,9 & Stricta ${ }^{a}$ & Yes & FM & Yes & $24 \mathrm{~h}$ & NPS (+) & NR & VS and UCB (-), AF (+) & NR & NR \\
\hline 8 & Dong L (7) & China & Female & No & $C D$ & 3,120 & 9,10 & NR & Yes & NR & Yes & $2 \mathrm{~h}$ & NPS (-) & $(+)$ & Vs (-) & NR & NR \\
\hline 9 & Oncel MY (33) & Turkey & Male & Yes (26w) & $C D$ & 1,010 & 2,6 & NR & Yes & FM & Yes & $1 d$ & DTA (+) & NR & NR & NR & $7 \mathrm{~d}$ \\
\hline 10 & Oncel MY (33) & Turkey & Male & Yes (33w) & VD & 1,950 & 7,8 & NR & Yes & FM & Yes & $5 d$ & NPS (+) & NR & NR & NR & $11 d$ \\
\hline 11 & Oncel MY (33) & Turkey & Female & No & $C D$ & 2,980 & 8,9 & NR & Yes & FM & Yes & $5 d$ & NPS (+) & NR & NR & NR & $7 \mathrm{~d}$ \\
\hline 12 & Oncel MY (33) & Turkey & Male & No & $C D$ & 3,700 & 8,9 & NR & Yes & FM & Yes & $2 d$ & NPS (+) & NR & NR & NR & $6 \mathrm{~d}$ \\
\hline 13 & Wu YT (34) & China & Female & No & $C D$ & 3,360 & 9,10 & Not strict & No & NR & Yes & NR & TS (+) & NR & NR & Asymptomatic & NR \\
\hline 14 & Wu YT (34) & China & Female & No & $C D$ & 3,570 & 9,10 & Not strict & No & NR & No & NR & TS (+) & $(-)$ & NR & $3 d$ & NR \\
\hline 15 & Wu YT (34) & China & Female & No & $C D$ & 2,760 & 10,10 & Not strict & No & NR & Yes & NR & TS (-) & $(+)$ & NR & Asymptomatic & NR \\
\hline 16 & Wu YT (34) & China & Female & No & $C D$ & 2,940 & 10,10 & Not strict & No & NR & Yes & NR & TS $(-)$ & NR & NR & Asymptomatic & NR \\
\hline 17 & Wu YT (34) & China & Female & No & $C D$ & 3,120 & 9,10 & Not strict & No & NR & Yes & $N R$ & TS $(-)$ & $(+)$ & NR & Asymptomatic & NR \\
\hline 18 & $\operatorname{HuX}(36)$ & China & Male & No & $C D$ & 3,250 & 8,10 & Strict $^{\mathrm{a}}$ & Yes & FM & No & $24-36 \mathrm{~h}$ & TS, feces and urine $(+)$ & NR & AF $(-)$ & Asymptomatic & NR \\
\hline
\end{tabular}

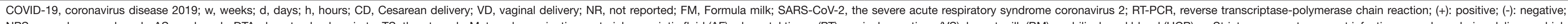

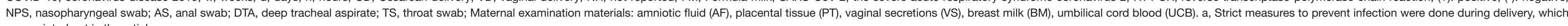
was pointed out in the article. 
artificial feeding. Therefore, most authors thought postnatal infection is less possible but could not be ruled out. For example, because Case 11 and 12 was diagnosed on the 5 th day of life (33), we cannot rule out the possibility of humanto-human transmission. Similarly, Case 13-17 failed to be isolated immediately from their mothers and were observed unprotected in the operating room for 30 minutes, thus the author believed that the contaminated air in the operating room may be a source of pollution (34).

What is different is Case 8 , who had elevated $\operatorname{IgG}$ and IgM antibody levels to SARS-CoV-2 and abnormal cytokine test results in blood 2 hours after birth but repeatedly negative RT-PCR tests results on nasopharyngeal swabs from 2 hours to 16 days after birth (7). This situation still meets the diagnostic criteria (3). The author Dong et al. thought that IgM antibodies usually do not appear until 3 to 7 days after infection and IgM antibodies cannot be transferred to the fetus via the placenta, thus the obviously elevated IgM in the neonate's blood sample drawn 2 hours after birth suggests that the neonate was infected in utero. IgG antibodies can be transmitted to the fetus through the placenta and appear later than IgM. Therefore, the elevated IgG level may reflect maternal or infant infection. In addition, the infant potentially could have been exposed for 23 days from the time of the mother's diagnosis of COVID-19 to delivery. Although amniotic fluid or placenta from the mother were not tested, but the evidence of vertical transmission was manifest in Case 8 . At this point, our views coincide with them. However, what confused us is the infant's repeatedly negative RT-PCR test results on nasopharyngeal swabs. If vertical transmission does occur in this case, this will prompt us: Unlike viruses that invade from the respiratory tract, viruses that invade from the placenta may not multiply in the nasopharynx.

Some cases also tested maternal examination materials for SARS-CoV-2 RT-PCR, such as amniotic fluid, placental tissue, mother's vaginal secretions, umbilical cord blood and breast milk. All the materials examined were negative for the virus except for amniotic fluid from Case 7 (21). The author Zamaniyan et al. thought the possibility of other transmission modes is small since they were sure that strict pollution control was done during and after delivery (21). They also suggested that the severity of COVID-19 of the mother is related to the neonate infection since the mother's condition was so severe that she died in the end. This author recorded the material collection process in detail (21), but subsequent repeated tests were not conducted and it was uncertain if the sample was polluted or not. Therefore, we believe that the possibility of false positives could not be ruled out and we cannot judge that whether vertical transmission occurred in Case 8.

In this systematic review, we failed to clarify the route of infection in SARS-Cov-2-positive neonates. The total infection rate of newborns to infected mothers is low, and we believe that the risk of vertical transmission is small. However, limited by the accuracy and completeness of the data, or some special circumstances (such as pregnant women receiving glucocorticoid therapy, fetuses with congenital diseases), we cannot exclude the possibility of vertical transmission of COVID-19. As clinical research continues to increase and deepen, more and more retrospective studies are required to explore this issue. Although we failed to obtain the definite evidence of vertical transmission of COVID-19, our study is still valuable as an alarm bell to recommend a more strict process of COVID-19 pregnant women's delivery.

\section{Acknowledgments}

The authors would like to thank the editors and the anonymous reviewers for insightful suggestions on this study. Funding: None.

\section{Footnote}

Reporting Checklist: The authors have completed the PRISMA reporting checklist. Available at http://dx.doi. org/10.21037/tp-20-144

Conflicts of Interest: All authors have completed the ICMJE uniform disclosure form (available at http://dx.doi. org/10.21037/tp-20-144). The authors have no conflicts of interest to declare.

Ethical Statement: The authors are accountable for all aspects of the work in ensuring that questions related to the accuracy or integrity of any part of the work are appropriately investigated and resolved.

Open Access Statement: This is an Open Access article distributed in accordance with the Creative Commons Attribution-NonCommercial-NoDerivs 4.0 International License (CC BY-NC-ND 4.0), which permits the noncommercial replication and distribution of the article with the strict proviso that no changes or edits are made and the original work is properly cited (including links to both the 
formal publication through the relevant DOI and the license). See: https://creativecommons.org/licenses/by-nc-nd/4.0/.

\section{References}

1. Gorbalenya AE, Baker SC, Baric RS, et al. Severe acute respiratory syndrome-related coronavirus: The species and its viruses - a statement of the Coronavirus Study Group. Nat Microbiol 2020;5:536-44.

2. Seymour CW, Liu VX, Iwashyna TJ, et al. Assessment of Clinical Criteria for Sepsis: For the Third International Consensus Definitions for Sepsis and Septic Shock (Sepsis-3). JAMA 2016;315:762-74.

3. National Health Commission of the People's Republic of China. New coronavirus pneumonia diagnosis and treatment program (Trial Version 8). 2020. Available online: http://www.nhc.gov.cn/

4. de Wit E, van Doremalen N, Falzarano D, et al. SARS and MERS: recent insights into emerging coronaviruses. Nat Rev Microbiol 2016;14:523-34.

5. Wong SF, Chow KM, Leung TN, et al. Pregnancy and perinatal outcomes of women with severe acute respiratory syndrome. Am J Obstet Gynecol 2004;191:292-7.

6. Alfaraj SH, Al-Tawfiq JA, Memish ZA. Middle East Respiratory Syndrome Coronavirus (MERS-CoV) infection during pregnancy: Report of two cases \& review of the literature. J Microbiol Immunol Infect 2019;52:501-3.

7. Dong L, Tian J, He S, et al. Possible Vertical Transmission of SARS-CoV-2 From an Infected Mother to Her Newborn. JAMA 2020;323:1846-8.

8. Moga C, Guo B, Schopflocher D, et al. Development of a Quality Appraisal Tool for Case Series Studies Using a Modified Delphi Technique. Edmonton AB: Institute of Health Economics 2012.

9. Xiong X, Wei H, Zhang Z, et al. Vaginal delivery report of a healthy neonate born to a convalescent mother with COVID--19. J Med Virol 2020;92:1657-9.

10. Mochizuki J, Nakamura M, Iwahata S, et al. First report in Japan of a delivery of a woman with the 2019 novel coronavirus disease. J Obstet Gynaecol Res 2021;47:407-10.

11. Wang X, Zhou Z, Zhang J, et al. A Case of 2019 Novel Coronavirus in a Pregnant Woman With Preterm Delivery. Clin Infect Dis 2020;71:844-6.

12. Huang L, Wang J, Xiong C, et al. Successful treatment of the first pregnancy with severe new coronavirus pneumonia. Med \& Pharm J Chin PLA 2020;32:1-4.
13. Yao L, Wang J, Zhao J, et al. Asymptomatic COVID-19 infection in pregnant woman in the third trimester: a case report. Chin J Perinat Med 2020;23:229-31.

14. Zhou R, Chen Y, Lin C, et al. Asymptomatic COVID-19 in pregnant woman with typical chest $\mathrm{CT}$ manifestation: a case report. Chin J Perinat Med 2020;23:E006.

15. Zhao Y, Lin Z, Mao X, et al. A case of dyspnea in newborns delivered by mothers infected with COVID-19. Chin J Neonatol 2020;35:84-5.

16. Chen S, Huang B, Luo DJ, et al. Pregnant women with new coronavirus infection: a clinical characteristics and placental pathological analysis of three cases. Chin J Pathol 2020;49:E005.

17. Li M, Xu M, Zhan W, et al. Report of the first cases of mother and infant infections with 2019 novel coronavirus in Xinyang City Henan Province. Chin J Infect Dis 2020;38:E007.

18. Zhuang S, Guo J, Cao Y, et al. Perinatal novel coronavirus infection: a case report. Chin J Perinat Med 2020;23:85-90.

19. Peng Z, Wang J, Mo Y, et al. Unlikely SARS-CoV-2 vertical transmission from mother to child: A case report. J Infect Public Health 2020;13:818-20.

20. Alzamora MC, Paredes T, Caceres D, et al. Severe COVID-19 during Pregnancy and Possible Vertical Transmission. Am J Perinatol 2020;37:861-5.

21. Zamaniyan M, Ebadi A, Aghajanpoor Mir S, et al. Preterm delivery in pregnant woman with critical COVID-19 pneumonia and vertical transmission. Prenat Diagn 2020. [Epub ahead of print].

22. Fan C, Lei D, Fang C, et al. Perinatal Transmission of COVID-19 Associated SARS-CoV-2: Should We Worry? Clin Infect Dis 2020. [Epub ahead of print].

23. Li Y, Zhao R, Zheng S, et al. Lack of Vertical Transmission of Severe Acute Respiratory Syndrome Coronavirus 2, China. Emerg Infect Dis 2020;26:1335-6.

24. Breslin N, Baptiste C, Gyamfi-Bannerman C, et al. Coronavirus disease 2019 infection among asymptomatic and symptomatic pregnant women: two weeks of confirmed presentations to an affiliated pair of $\mathrm{New}$ York City hospitals. Am J Obstet Gynecol MFM 2020;2:100118.

25. Liu W, Wang J, Li W, et al. Clinical characteristics of 19 neonates born to mothers with COVID-19. Front Med 2020;14:193-8.

26. Chen Y, Peng H, Wang L, et al. Infants Born to Mothers With a New Coronavirus (COVID-19). Front Pediatr 2020;8:104.

27. Chen S, Liao E, Cao D, et al. Clinical analysis of pregnant 
women with 2019 novel coronavirus pneumonia. J Med Virol 2020;92:1556-61.

28. Yu N, Li W, Kang Q, et al. Clinical features and obstetric and neonatal outcomes of pregnant patients with COVID-19 in Wuhan, China: a retrospective, single-centre, descriptive study. Lancet Infect Dis 2020;20:559-64.

29. Zeng L, Xia S, Yuan W, et al. Neonatal Early-Onset Infection With SARS-CoV-2 in 33 Neonates Born to MothersWithCOVID-19 in Wuhan, China. JAMA Pediatr 2020;174:722-5.

30. Zhu H, Wang L, Fang C, et al. Clinical analysis of 10 neonates born to mothers with 2019-nCoV pneumonia. Transl Pediatr 2020;9:51-60.

31. Prabhu M, Cagino K, Matthews KC, et al. Pregnancy and postpartum outcomes in a universally tested population for SARS-CoV-2 in New York City: a prospective cohort study. Bjog 2020;127:1548-56.

32. Salvatore CM, Han JY, Acker KP, et al. Neonatal

Cite this article as: Yuan J, Qian H, Cao S, Dong B, Yan X, Luo S, Zhou M, Zhou S, Ning B, Zhao L. Is there possibility of vertical transmission of COVID-19: a systematic review. Transl Pediatr 2021;10(2):423-434. doi: 10.21037/tp-20-144 management and outcomes during the COVID-19 pandemic: an observation cohort study. Lancet Child Adolesc Health 2020;4:721-7.

33. Oncel MY, Akın IM, Kanburoglu MK, et al. A multicenter study on epidemiological and clinical characteristics of 125 newborns born to women infected with COVID-19 by Turkish Neonatal Society. Eur J Pediatr 2020:1-10. [Epub ahead of print].

34. Wu Y'T, Liu J, Xu JJ, et al. Neonatal outcome in 29 pregnant women with COVID-19: A retrospective study in Wuhan, China. PLoS Med 2020;17:e1003195.

35. Yan J, Guo J, Fan C, et al. Coronavirus disease 2019 in pregnant women: a report based on 116 cases. Am J Obstet Gynecol 2020;223:111.e1-111.e14.

36. Hu X, Gao J, Luo X, et al. Severe Acute Respiratory Syndrome Coronavirus 2 (SARS-CoV-2) Vertical Transmission in Neonates Born to Mothers With Coronavirus Disease 2019 (COVID-19) Pneumonia. Obstet Gynecol 2020;136:65-7. 